\title{
Hibernoma - two patients with a rare lipoid soft-tissue tumour
}

\author{
Dirk Daubner ${ }^{1 *}$, Stephanie Spieth², Jessica Pablik³ ${ }^{3}$ Klaus Zöphel ${ }^{4}$, Tobias Paulus $^{2}$ and Michael Laniado ${ }^{2}$
}

\begin{abstract}
Background: Hibernomas are rare benign soft-tissue tumours arising from brown fat tissue. Although imaging characteristics are not specific certain imaging features, common locations and patient demographics may suggest hibernoma as a differential diagnosis.

Case presentation: We report on two 48-year-old male patients with hibernoma. The tumour presented with local swelling of the inguinal region in the first patient and was an incidental imaging finding in the second patient. Imaging included magnetic resonance imaging in both patients and computed tomography as well as ${ }^{18} \mathrm{~F}$-fluorodeoxyglucose positron emission tomography-computed tomography in the second patient. In both cases histological diagnosis was initially based on excisional and needle core biopsy, respectively. Complete surgical resection confirmed the diagnosis of hibernoma thereafter.
\end{abstract}

Conclusion: In soft tissue tumours with fatty components hibernoma may be included into the differential diagnosis. Because of the risk of sampling errors in hibernoma-like tissue components of myxoid and well-differentiated liposarcoma, complete resection is mandatory. This article also reviews the current imaging literature of hibernomas.

Keywords: Hibernoma, Brown fat tissue, Soft-tissue tumour, Liposarcoma, MRI, ${ }^{18}$ F-FDG-PET/CT

\section{Background}

Hibernomas are rare benign soft-tissue tumours arising from brown fat tissue [1-3]. The tumour was first described in 1906 by Merkel who named them "pseudolipoma" [4]. The name hibernoma was given by Gery in 1914 because of its similarity to brown fat in hibernating animals [5]. Hibernomas count for approximately 1.6\% of all benign lipomatous tumours [6]. Until now, case reports and series with up to 170 patients were published [7]. Males are more often involved than females [7]. The typical age at presentation is between 30 and 50 years. Hibernomas are slowly growing, painless tumours, either presenting as palpable soft and mobile mass or as an incidental finding at imaging [3]. Due to the hypervascularity, hibernomas may sometimes appear with locally increased skin temperature. They can reach a size of up to $20 \mathrm{~cm}$ so that symptoms secondary to compression of adjacent structures may developed [7].

\footnotetext{
* Correspondence: Dirk.Daubner@uniklinikum-dresden.de

'Department of Neuroradiology, Carl Gustav Carus Medical School, University of Dresden, Fetscherstraße 74, 01307 Dresden, Germany

Full list of author information is available at the end of the article
}

The aetiology of hibernomas is unknown. Molecular genetics show deletions respectively reciprocal translocation of chromosome 11 which encodes the tumour suppressor gene MEN $1[8,9]$. The development of hibernomas was initially thought to be related to areas of residual brown fat such as the interscapular area, axilla, chest wall, mediastinum or retroperitoneum. However, single case reports and a clinical-pathological study with 170 cases showed the thigh to be a preferential location with about $30 \%$ of the cases [7].

At histopathology, most hibernomas present as typical variant $(82 \%)$ of which three cellular subtypes are differentiated (pale staining, mixed and eosinophilic cells). Myxoid variants (9\%), lipoma-like variants (7\%), and spindle cell variants (2\%) of hibernoma are less common [3,7].

Except intrathoracic and retroperitoneal hibernomas, the tumours are usually well diagnosed with ultrasound showing smooth borders and hyperechoic signal. Colour duplex sonography and angiography often present a hypervascular tumour, with arteriovenous shunts in some cases. Due to the increased risk of bleeding, biopsy is not recommended in these cases. But often the diagnosis is not considered prior to biopsy in different imaging 
modalities, as presented in the two following cases $[3,10,11]$.

In computed tomography $(\mathrm{CT})$ and magnetic resonance imaging (MRI), hibernomas are well-circumscribed tumours, commonly located in subcutaneous tissue, skeletal muscle or intermuscular fascial planes. Common sites include thigh, peri- and interscapular region, axilla, neck, chest, abdominal cavity and retroperitoneum. The scalp, breast, scrotum and perirectal region, spine and bones are less frequently affected $[3,7,12]$. Hibernomas show relatively low attenuation in CT between the attenuation of subcutaneous fat and skeletal muscle. Intratumoural septa and a variable degree of enhancement may be seen $[1,13,14]$. In MRI, hibernomas may be slightly hypointense to fatty tissue on T1- and T2-weighted images and markedly hyperintense when fat suppressed T2-weighted inversion recovery sequences are applied. Elongated and branching vessels within the tumour are typically seen as flow void phenomena in MRI. Nevertheless, lack of intratumoural vessels does not exclude hibernoma [2,3,10]. After intravenous contrast agent administration strong enhancement of intratumoural blood vessels and homogeneous or heterogeneous tumour enhancement may be observed [10]. In ${ }^{18}$ F-fluorodeoxyglucose (FDG) positron emission tomography-computed tomography (PET/CT) high uptake is measured in hibernomas due to the metabolic activity of brown fat. Therefore, differentiation from malignant tumours is difficult $[3,10,15,16]$. Compared to liposarcomas, hibernomas often show higher standardized uptake values (SUVs) and change of FDG avidity in follow-up. However, these criteria are not reliable enough to make the diagnosis based on imaging alone $[16,17]$.
Hibernomas are benign tumours without a risk of malignant transformation or metastases $[7,18]$. As soon as the diagnosis is confirmed, no surgical removal or other treatment is required in asymptomatic hibernoma [16]. However, histological features similar to hibernomas are described in atypical fatty tumours as well as in myxoid and well-differentiated liposarcomas. Consequently, biopsy proven diagnosis of hibernoma does not exclude hibernoma-like differentiation in myxoid or welldifferentiated liposarcomas and complete excision is indicated. Incomplete resection of hibernoma carries the risk of recurrence, whereas complete resection cures the disease $[7,19]$.

\section{Case presentation}

Case 1

A 48-year-old male patient was admitted to our hospital with a palpable soft-tissue swelling at the left upper leg extending to the groin. The tumour was slowly growing since approximately 6 months. MRI was performed (Siemens Magnetom Verio ${ }^{\circ}$, 3.0 Tesla, body-array-coil, Erlangen, Germany) showing a $4 \times 10 \times 12 \mathrm{~cm}$ measuring mass between the gluteus medius and minimus muscles which was isointense to fat in all sequences. The tumour extended from the greater trochanter of the femur to the anterior superior iliac spine (Figure 1). After intravenous administration of contrast medium (15 ml Magnevist ${ }^{\circ}, 0.5 \mathrm{mmol} / \mathrm{ml}$, Bayer Vital GmbH, Leverkusen, Germany) slight rim enhancement of the tumour (arrow in Figure 1c) and a prominent vessel within the tumour (arrow in Figure 1d) were seen. Based on imaging findings, the working diagnosis was

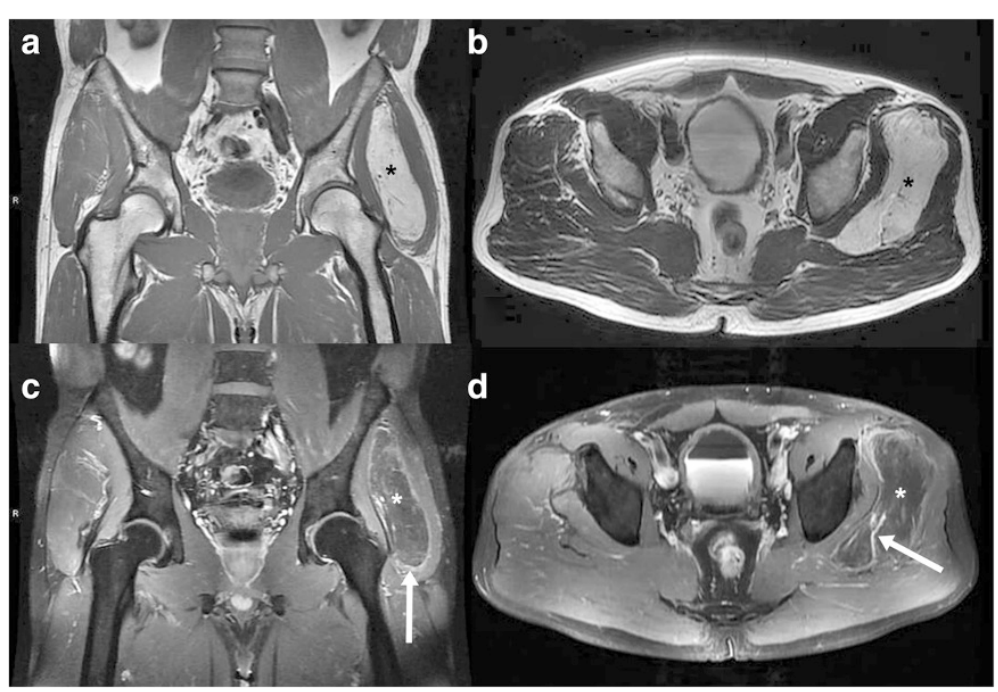

Figure 1 48-year-old man with hibernoma of the left upper leg. Tumour between the gluteus medius and minimus muscles (star in image 1 a-d). MRI shows an isointense tumour compared to fat with slight rim enhancement (arrow in c) and a prominent vessel within the mass (arrow in $\mathbf{d}$ ). a plain T1-weighted-TSE (TR 819 ms/ TE 11 ms), b plain T2-weighted-TSE (TR 5050 ms/ TE 96 ms), c contrast enhanced fat saturated T1-weighted-TSE (TR 895 ms/ TE 11 ms), d contrast enhanced fat saturated T1-weighted-TSE (TR 680 ms/ TE 11 ms). 


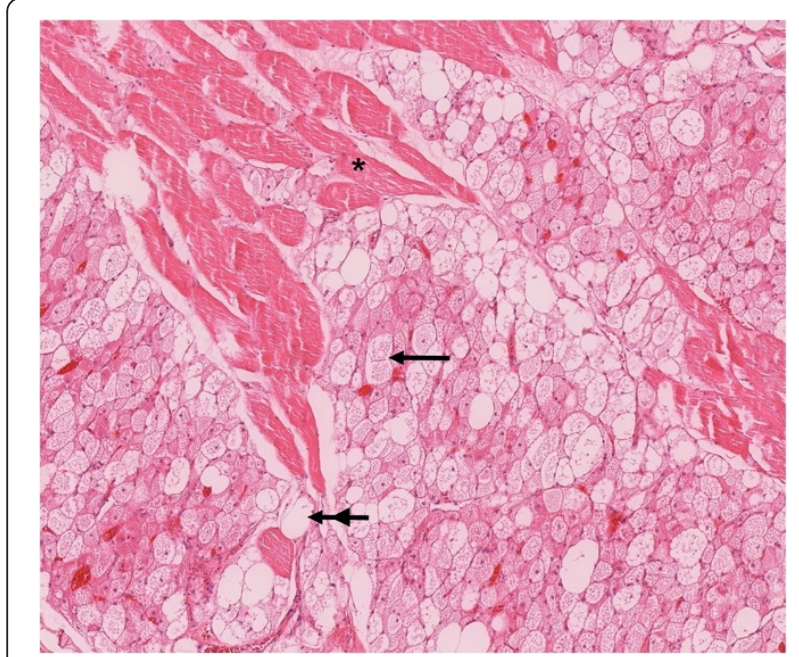

Figure 2 Tumour, composed of multivacuolated eosinophilic (arrow) and pale cells (double arrow), dissecting between skeletal muscle fibers (asterisk). $\mathrm{H}$ \& $\mathrm{E}$ stain, original magnification 10x.

liposarcoma. The case was discussed in the multidisciplinary sarcoma board of our comprehensive cancer center. As a result, diagnostic excision was suggested which was performed without complications.

Histopathology of the specimen $(2 \times 1.2 \times 1 \mathrm{~cm})$ revealed an intramuscular lipomatous neoplasia, complying with the diagnosis of the pale cell subtype of typical hibernoma (Figure 2). In-toto excision of the encapsulated hibernoma followed two weeks later. Histopathology confirmed the diagnosis of hibernoma and liposarcoma could be excluded. Postsurgery MRI follow-up after one year proved that no recurrence has occurred.

\section{Case 2}

A 48-year-old male patient presented in our emergency department with upper abdominal pain, elevated liver enzymes and laboratory parameters of cholestasis. Acute cholecystitis was suspected and abdominal ultrasound was performed. Due to overlying bowel gas the gallbladder could not be examined completely. Consequently, abdominal CT (Siemens Somatom Definition AS + ${ }^{\circ}$, Erlangen, Germany, collimation $2 \times 64 \times 0.6 \mathrm{~mm}$, current $129 \mathrm{~mA}$, tube voltage $120 \mathrm{kV}$ ) with both intravenous (90 ml Solutrast $300 \mathrm{mg} \mathrm{I/ml,} \mathrm{Bracco} \mathrm{Imaging} \mathrm{Deutschland}$ $\mathrm{GmbH}$, Konstanz, Germany; imaging delay $65 \mathrm{sec}$.) and oral contrast administration $\left(1000 \mathrm{ml} \mathrm{H}_{2} 0+30 \mathrm{ml}\right.$ Gastrolux ${ }^{\circ}$ 370 mg I/ml, Sanochemia Diagnostics Deutschland GmbH, Neuss, Germany) was performed to exclude gallbladder perforation. CT showed increased enhancement of the gallbladder wall and pericholecystitic infiltration of fatty tissue compatible with acalculous cholecystitis. There was no dilatation or wall thickening of the intra- or extrahepatic bile

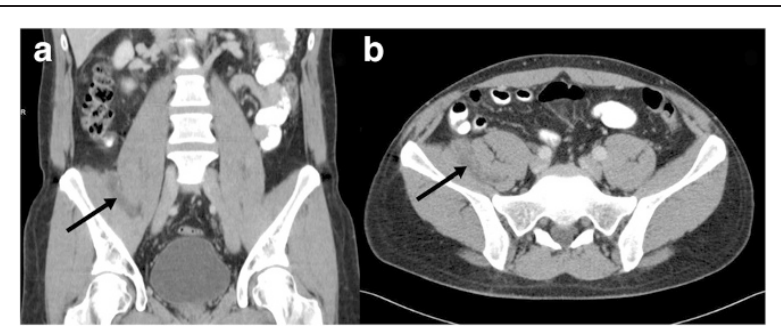

Figure 3 48-year-old man with right-sided retroperitoneal hibernoma. Tumour between the psoas and iliac muscles (arrows). Contrast enhanced $C T$ shows minimal heterogeneous enhancement of the tumour. a coronal reconstruction, $\mathbf{b}$ axial slice.

ducts. Endosonography was performed thereafter, showing a single stone in the middle part of the common bile duct, which was treated with endoscopic retrograde cholangiopancreatography.

CT incidentally revealed a $5.5 \times 1.5 \times 5.5 \mathrm{~cm}$ measuring retroperitoneal tumour located between the right psoas and iliac muscles (Figure 3). The tumour showed mean density values between 10-15 Hounsfield Units (HU). For further diagnostic work-up MRI of the abdomen was performed (Siemens Magnetom Verio', 3.0 Tesla, body-array-coil, Erlangen, Germany). Turbo inversion recovery magnitude (TIRM) sequences revealed a hyperintense tumour that was slightly hypointense in T1- and T2-weighted sequences compared to mesenteric fat. After administration of intravenous contrast agent $(15 \mathrm{ml}$ Magnevist ${ }^{\circ}, 0,5 \mathrm{mmol} / \mathrm{ml}$, Bayer Vital $\mathrm{GmbH}$, Leverkusen, Germany) there was a strong heterogeneous enhancement. Based on CT and MRI the working diagnosis was a potentially malignant mesenchymal tumour (Figure 4). Therefore, PET/CT (Siemens Biograph $16^{\circ}$, Knoxville, USA, collimation $16 \times 1.5 \mathrm{~mm}$, tube currency $9 / 97 \mathrm{mAs}$, tube voltage $100 / 120 \mathrm{kV}$ ) with ${ }^{18} \mathrm{~F}$-FDG (5 MBq/kg body weight) was performed which showed an increased accumulation of ${ }^{18}$ F-FDG $\left(\mathrm{SUV}_{\max } 12.3\right)$ within the tumour 60 minutes after injection. ${ }^{18} \mathrm{~F}$-FDG-PET/CT further supported the working diagnosis of a malignant tumour (Figure 5).

Histological examination was performed after CTguided biopsy of the soft-tissue lesion (Quick Core Biopsy Needle Sets QCS-18-15-20 T, Cook Medical Inc., Bloomington, USA). Histological analysis yielded the pale cell subtype of a typical hibernoma (Figure 6). After discussion in the multidisciplinary sarcoma board of the comprehensive cancer center at our hospital elective tumour excision was performed 2 months after biopsy. The final histological diagnosis of the surgical specimen confirmed the presumptive diagnosis of the biopsy.

\section{Conclusion}

Hibernomas are benign, well-circumscribed, slowly growing and well vascularised soft-tissue tumours containing 


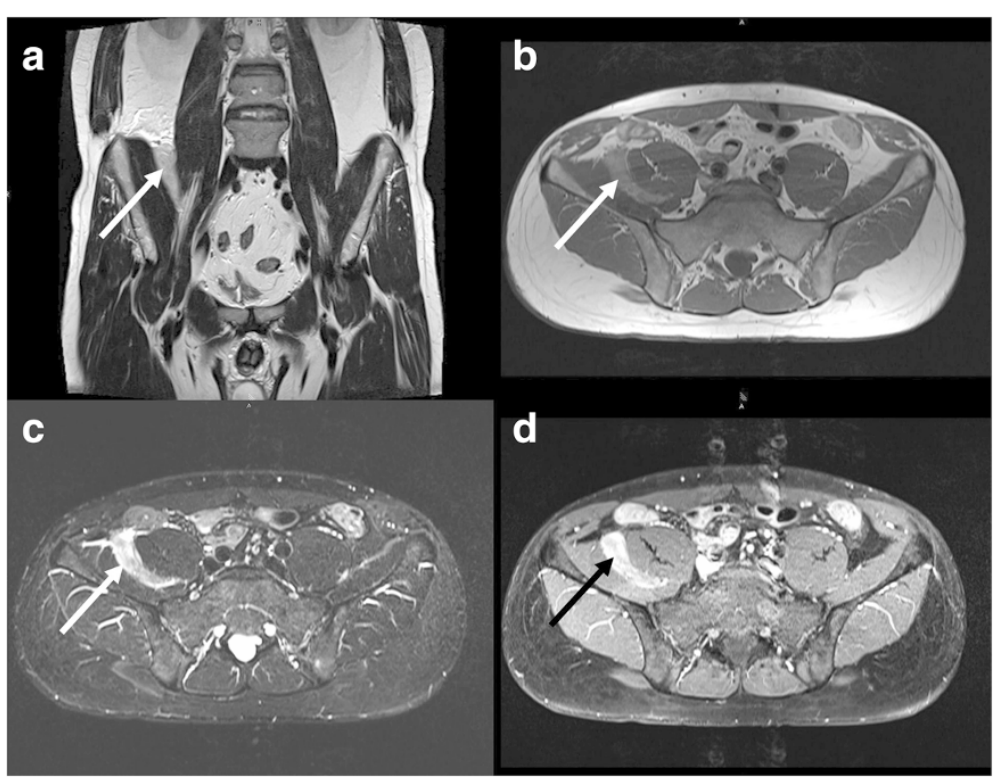

Figure 4 MRI of the same patient as shown in Figure 3. The tumour (arrows) is slightly hypointense compared to mesenteric fat in T2- (a, TSE, TR 5040 ms/ TE 137 ms) and T1-weighted images (b, TSE, TR 651 ms/ TE 11 ms). The T2-weighted TIRM sequence (c) shows marked hyperintensity of the mass (c, TR 5000 ms/ TE 74 ms/ TI 170 ms). After administration of contrast medium heterogeneous enhancement occurs (d, fat saturated TSE, TR 600 $\mathrm{ms} / \mathrm{TE} 11 \mathrm{~ms})$.

brown fat [1-3,7]. MRI and ${ }^{18}$ F-FDG-PET may provide imaging findings suggestive of hibernoma. These findings include hypointensity to fatty tissue on T2-weighted images and hyperintense signal intensity on TIRM images. Large intratumoural blood vessels, heterogeneous contrast enhancement and strong FDG uptake are further imaging signs $[2,3,10,15,16]$.

The differential diagnosis of lipomatous soft-tissue tumours is broad and comprises benign (e. g. lipoma, hemangioma, angiolipoma) and malignant (e. g. liposarcoma) lesions $[1,14,20]$. Lipomas are characterized by homogeneous adipose tissue which can show thin septa. There is no contrast enhancement within lipomas, although infections, infarctions and necrosis as well as muscle fibres, intratumoural vessels und fibrotic septa may hamper the diagnosis [21]. Dependent on fat content,

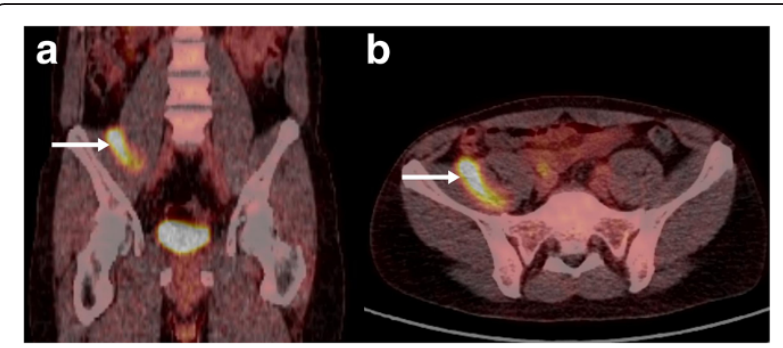

Figure $5{ }^{18} \mathrm{~F}$-FDG-PET/CT of the same patient as shown in Figure 3 and 4 demonstrates increased FDG-activity of the mass (arrows). CT was performed in low-dose technique ( $9 / 97 \mathrm{mAs}$, 100/120 kV). a coronal reconstruction, b axial slice. haemangiomas and angiolipomas show hypo- to hyperintense signal on T1-weighted images and hyperintense signal on T2-weighted images with intrinsic vessels. Haemangiomas can also contain calcifications similar to phlebolits [10]. Imaging characteristics of liposarcomas depends on the histological differentiation. Well-differentiated liposarcomas have thickened septa $(>2 \mathrm{~mm})$ as well as areas of T2-hyperintensity. Enhancement can be absent or circumscribed $[10,20,21]$. The signal intensity on T1-weighted images is often similar to subcutaneous fat. Moreover, large

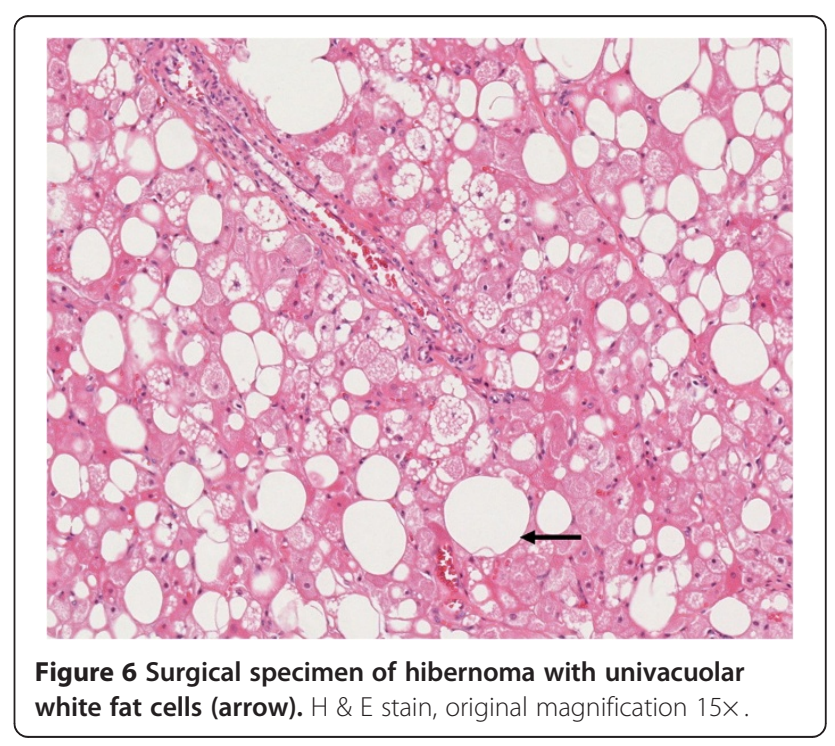


Table 1 Summary table of hibernomas

\begin{tabular}{ll}
\hline Aetiology & Unclear, deletion respectively reciprocal translocation of chromosome 11 \\
Incidence & Approximately 1.6\% of all benign lipomatous tumours \\
Gender distribution & Slight male predominance \\
Average age & 3rd to 4th decade of life \\
Risk factors & Not known \\
Therapy & Complete excision of symptomatic hibernoma or unclear diagnosis \\
Prognosis & Benign tumour without malignant transformation or metastatic spread \\
& $\begin{array}{l}\text { No recurrence after complete excision, possible recurrence in cases of incomplete excision } \\
\text { Imaging characteristics }\end{array}$ \\
& $\begin{array}{l}\text { Well-defined, richly vascularized soft tissue tumour with a density between subcutaneous fat and skeletal muscle in CT, } \\
\text { respectively slightly hypointense to subcutaneous fat in T1w and T2W MR images, hyperintense in TIRM, heterogenous to } \\
\text { homogenous enhancement, high }{ }^{18} \mathrm{~F}-\mathrm{FDG} \text { uptake and change of FDG avidity in follow-up }\end{array}$ \\
\hline
\end{tabular}

vessels are lacking which helps to distinguish liposarcomas from hibernomas. Poorly differentiated liposarcomas are rarely confounded with hibernomas due to the low fat content $[20,21]$.

However, despite the use of multimodal imaging, the radiologic diagnosis of hibernomas is difficult and differentiation from well-differentiated liposarcomas remains a challenge even after biopsy. Therefore, complete surgical resection is highly recommended. A summary table of hibernomas is shown in Table 1.

\section{Consent}

Written informed consent was obtained from both patients for publication of this Case report and any accompanying images. A copy of the written consents is available for review by the Editor of this journal.

\section{Abbreviations \\ CT: Computed tomography; MRI: Magnetic resonance imaging; FDG: Fluorodeoxyglucose; PET: Positron emission tomography; TIRM: Turbo inversion recovery magnitude; HU: Hounsfield units; SUV: Standardized uptake value.}

\section{Competing interests}

The authors declare that they have no competing interests.

\section{Authors' contributions}

DD - conception, design and manuscript preparation. SS - manuscript preparation and English translation. JP - contribution of the pathological part. KZ - contribution of the nuclear medicine part. TP - conduction and analysis of exams. $\mathrm{ML}$ - made substantial contribution to the concept of the manuscript, final correction of the manuscript with respect to important intellectual content. All authors read and approved the manuscript.

\section{Acknowledgments}

The authors gratefully acknowledge the support of Prof. Dr. Rüdiger von Kummer (Department of Neuroradiology, University of Dresden) and Dr. Benita Wolf (École polytechnique fédérale de Lausanne) for reviewing the manuscript.

\section{Author details}

'Department of Neuroradiology, Carl Gustav Carus Medical School, University of Dresden, Fetscherstraße 74, 01307 Dresden, Germany. ${ }^{2}$ Department of Radiology, Carl Gustav Carus Medical School, University of Dresden, Fetscherstraße 74, 01307 Dresden, Germany. '3epartment of Pathology, Carl Gustav Carus Medical School, University of Dresden, Fetscherstraße 74, 01307 Dresden, Germany. ${ }^{4}$ Department of Nuclear Medicine, Carl Gustav Carus Medical School, University of Dresden, Fetscherstraße 74, 01307 Dresden, Germany.
Received: 14 May 2014 Accepted: 2 February 2015

Published online: 14 February 2015

\section{References}

1. Anderson SE, Schwab C, Stauffer E, Banic A, Steinbach LS. Hibernoma: imaging characteristics of a rare benign soft tissue tumor. Skeletal Radiol. 2001;30:590-5.

2. Liu W, Bui MM, Cheong D, Caracciolog JT. Hibernoma: comparing imaging appearance with more commonly encountered benign or low-grade lipomatous neoplasms. Skeletal Radiol. 2013;42:1073-8.

3. Murphey MD, Carroll JF, Flemming DJ, Pope TL, Gannon FH, Kransdorf MJ. From the archives of the AFIP: benign musculoskeletal lipomatous lesions. Radiographics. 2004;24:1433-66.

4. Merkel H. On a pseudolipoma of the breast. Beitr Pathol Anat. 1906:39:152-7.

5. Gery L. In discussion of MF Bonnel's paper. Bull Mem Soc Anat (Paris). 1914;89:111-2

6. Miettinen MM, Fanburg-Smith JC, Mandahl N. Hibernoma. In: Fletscher CDM, Unni KK, Mertens F, editors. Pathology and Genetics of Tumours of Soft Tissue and Bone. Lyon, France: IARC Press; 2002. p. 19-46.

7. Furlong MA, Fanburg-Smith J, Miettinen M. The morphologic spectrum of hibernoma: a clinicopathologic study of 170 cases. Am J Surg Pathol. 2001;25:809-14.

8. Mertens F, Rydholm A, Brosjö O, Willén H, Mitelman F, Mandahl N. Hibernomas are characterized by rearrangements of chromosome bands 11q13-21. Int J Canver. 1994;58:503-5.

9. Mrózek K, Karakousis CP, Bloomfield CD. Band 11q13 is nonrandomly rearranged in hibernomas. Genes Chrom Cancer. 1994:9:145-7.

10. Papathanassiou ZG, Alberghini M, Taieb S, Errani C, Picci P, Vanel D. Imaging of hibernomas: a retrospective study on twelve cases. Clin Sarcoma Res. 2011;1(3):1-11.

11. Hardes J, Scheil-Bertram S, Hartwig E, Gebert C, Gosheger G, Schulte M. Sonographic findings of hibernomas. A report of two cases. J Clin Ultrasound. 2005:33:298-301

12. Bonar SF, Watson G, Gragnaniello C, Seex K, Magnussen J, Earwaker J. Intraosseous hibernoma: characterization of five cases and literature review. Skeletal Radiol. 2014;43:939-46

13. Dursun M, Agayev A, Bakir B, Ozger H, Eralp L, Sirvanci M, et al. CT and MR characteristics of hibernoma: six cases. Clin Imaging. 2008:32:42-7.

14. Atilla S, Eilenberg SS, Brown JJ. Hibernoma: MRI appearance of a rare tumor. Magn Reson Imaging. 1995;13:335-7.

15. Chatterton BE, Mensforth D, Coventry BJ, Cohen P. Hibernoma: intense uptake seen on Tc-99 m tetrofosmin and FDG positron emission tomographic scanning. Clin Nucl Med. 2002;27:369-70.

16. Schmidt F, Cathomas R, Stallmach T, Putora PM, Mueller J. Have you ever heard of hibernoma? A rare but important pitfall in FDG-PET/CT. Nuklearmedizin. 2010;49:N71-3.

17. Smith S, Teruya-Feldstein J, Caravelli JF, Yeung HW. False-positive findings on ${ }^{18}$ F-FDG PET/CT: differentiation of hibernoma and malignant fatty tumor on the basis of fluctuating standardized uptake values. Am J Roentgenol. 2008;190:1091-6. 
18. Enterline HT, Lowry LD, Richman AV. Does malignant hibernoma exist? Am J Surg Pathol. 1979;3:265-71.

19. Lele SM, Chundru S, Chaljub G, Adegboyega P, Haque AK. Hibernoma: a report of 2 unusual cases with a review of the literature. Arch Pathol Lab Med. 2002;126:975-8.

20. Lee JC, Gupta A, Saifuddin A, Flanagan A, Skinner JA, Briggs TWR, et al. Hibernoma: MRI features in eight consecutive cases. Clin Radiol. 2006:61:1029-34.

21. Gaskin CM, Helms CA. Lipomas, lipoma variants, and well-differentiated liposarcomas (atypical lipomas): results of MRI evaluations of 126 consecutive fatty masses. Am J Roentgenol. 2004;182:733-9.

\section{Submit your next manuscript to BioMed Central} and take full advantage of:

- Convenient online submission

- Thorough peer review

- No space constraints or color figure charges

- Immediate publication on acceptance

- Inclusion in PubMed, CAS, Scopus and Google Scholar

- Research which is freely available for redistribution 